\title{
Clinical Manifestations and Risk Factors for Complications of Philadelphia Chromosome-Negative Myeloproliferative Neoplasms
}

\author{
Boonlerd Duangnapasatit ${ }^{1}$, Ekarat Rattarittamrong ${ }^{2 *}$, Thanawat \\ Rattanathammethee $^{2}$, Sasinee Hantrakool ${ }^{2}$, Chatree Chai-Adisaksopha ${ }^{2}$, Adisak \\ Tantiworawit ${ }^{2}$, Lalita Norasetthada ${ }^{2}$
}

\begin{abstract}
Background: Myeloproliferative neoplasms (MPNs) are clonal hematopoietic stem cell disorders characterized by proliferation of one or more myeloid lineages. Polycythemia vera (PV), essential thrombocythemia (ET) and primary myelofibrosis (PMF) are classical Philadelphia chromosome (Ph)-negative MPN that have a Janus Kinase 2 (JAK2) mutation, especially JAK2V617F in the majority of patients. The major complications of Ph-negative MPNs are thrombosis, hemorrhage, and leukemic transformation. Objective: To study clinical manifestations including symptoms, signs, laboratory findings, and JAK2V617F mutations of Ph-negative MPN (PV, ET and PMF) as well as their complications. Materials and Methods: All Ph-negative MPN (PV, ET and PMF) patients who attended the Hematology Clinic at Maharaj Nakorn Chiang Mai Hospital from January, 1 2003 through December, 312013 were retrospectively reviewed for demographic data, clinical characteristics, complete blood count, JAK2V617F mutation analysis, treatment, and complications. Results: One hundred and fifty seven patients were included in the study. They were classified as PV, ET and PMF for 68,83 and 6 with median ages of 60,61 , and 68 years, respectively. JAK2V617F mutations were detected in $88 \%, 69 \%$, and $100 \%$ of PV, ET and PMF patients. PV had the highest incidence of thrombosis (PV 29\%, ET 14\%, and PMF 0\%) that occurred in both arterial and venous sites whereas PMF had the highest incidence of bleeding (PMF 17\%, ET 11\%, and PV 7\%). During follow up, there was one ET patient that transformed to acute leukemia and five cases that developed thrombosis (three ET and two PV patients). No secondary myelofibrosis and death cases were encountered. Conclusions: Ph-negative MPNs have various clinical manifestations. JAK2V617F mutations are present in the majority of PV, ET, and PMF patients. This study confirmed that thrombosis and bleeding are the most significant complications in patients with Ph-negative MPN.
\end{abstract}

Keywords: Myeloproliferative neoplasms - polycythemia vera - essential thrombocythemia - primary myelofibrosis

Asian Pac J Cancer Prev, 16 (12), 5013-5018

\section{Introduction}

Myeloproliferative neoplasms (MPNs) are clonal hematopoietic stem cell disorders characterized by the proliferation of one or more myeloid lineages. Classical MPNs include chronic myelogenous leukemia (CML), polycythemia vera (PV), essential thrombocythemia (ET) and primary myelofibrosis (PMF) (Tefferi and Vardiman, 2008). CML has a distinct pathogenesis due to the presence of a Philadelphia chromosome (Ph) or $B C R-A B L 1$ fusion gene. PV,ET, and PMF are so called "Ph-negative MPNs" and have a mutation of Janus Kinase 2 (JAK2) gene, commonly $J A K 2 \mathrm{~V} 617 \mathrm{~F}$ mutation, in the majority of patients (Tefferi and Vardiman, 2008; Barbui et al., 2011).

Although Ph-negative MPNs share the same mutation, they have different clinical manifestations and laboratory findings. PV is characterized by the proliferation of all myeloid lineages whereas ET has predominantly megakaryocyte proliferation and thrombocytosis. In the PMF case, cytokines from abnormal megakaryocytes lead to fibrosis in bone marrow and extramedullary hematopoiesis (Tefferi A, 2008). The diagnosis of Phnegative MPNs required clinical data, complete blood count (CBC), and bone marrow histology. JAK2V617F mutation analysis was also helpful for the diagnosis, however, it was not detected in all $\mathrm{Ph}$-negative MPNs patients. More than $90 \%$ of PV patients had a JAK2V617F mutation and more than half of the ET and PMF cases harbored this mutation (Tefferi et al., 2008; Barbui et al.; 2011; Tefferi et al., 2013).

The major complication of Ph-negative MPNs especially in PV and ET was thrombosis. The incidence of

${ }^{I}$ Department of Internal Medicine, ${ }^{2}$ Division of Hematology, Department of Internal Medicine, Faculty of Medicine, Chiang Mai University, Chiang Mai, Thailand *For correspondence: ekarat.r@cmu.ac.th,ekarat_r@hotmail.com 
thrombosis in PV was $23.4 \%$ from an international study of PV (Tefferi et al., 2013). The risk factors of thrombosis from the prospective study were an age more than 65 years and a history of thrombosis (Marchioli, 2005). In ET, the International Prognostic Score of thrombosis in World Health Organization-Essential Thrombocythemia (IPSET)-thrombosis study showed a $12 \%$ incidence of thrombosis (Carobbio et al., 2011). The risk factors of thrombosis were age more than 60 years, cardiovascular risk factors (diabetes mellitus, hypertension, and smoking), leukocytosis more than $11 \times 10^{9} / \mathrm{L}$, and the presence of a JAK2V617F mutation (Carobbio et al., 2011; Barbui et al., 2012). Other complications were hemorrhage, leukemic transformation and secondary myelofibrosis in cases of PV and ET (Tefferi et al., 2008; Barbui et al., 2011).

The current treatment for PV includes phlebotomy for maintaining hemoglobin $(\mathrm{Hb})$ levels less than $45 \%$, low dose aspirin, as well as controlling cardiovascular risk factors. Cytoreductive therapy such as hydroxyurea is indicated in patients with a high risk of thrombosis. Similarly for ET, low dose aspirin, cardiovascular risk factor management, and cytoreductive therapy in cases of high risk of thrombosis were also used. Aspirin however, should be avoided in patients with extreme thrombocytosis (platelet more than $1,000 \times 10^{9} / \mathrm{L}$ ) who have acquired von Willibrand disease. For the treatment of PMF, most patients received symptom-based therapy for instance, blood transfusion in cases of anemia and hydroxyurea in cases of splenomegaly. Allogeneic stem cell transplantation should be considered in young patients with high risk features (Barbui et al., 2011).

The primary objective of this study aimed to study clinical manifestations including symptoms, signs, laboratory findings, and JAK2V617F mutation of Ph-negative MPN (PV, ET and PMF) as well as their complications. The secondary objective was to determine the risk factors of thrombosis and bleeding in $\mathrm{Ph}$-negative MPN patients.

\section{Materials and Methods}

\section{Study overview}

This retrospective cohort study was approved by the Institutional Review Board (IRB) of the Faculty of Medicine, Chiang Mai University, Thailand and performed at Maharaj Nakorn Chiang Mai Hospital. All Ph-negative MPN (PV, ET and PMF) patients who attended the Hematology Clinic from January, 12003 through December, 312013 were retrospectively reviewed for demographic data (age, sex, underlying diseases), clinical characteristics (signs and symptoms including splenomegaly), CBC at the diagnosis, JAK2 V617F mutation analysis, treatment, and complications (thrombosis, hemorrhage, secondary myelofibrosis, and leukemic transformation). Exclusion criteria were patients with underlying hematologic diseases that interferred with their CBC. The survival risk scores from the International Study of PV (age, WBC count more than $15 \times 109 / \mathrm{L}$, and venous thrombosis) (Tefferi et al., 2013) were calculated in patients with PV. The International Prognostic Score for ET-thrombosis (IPSET-thrombosis) (Carobbioet al.,
2011; Barbui et al., 2012) were evaluated in ET patients for risk determination of thrombosis. For PMF patients, the International Prognosis Scoring System (IPSS) (Cervantes et al., 2009), Dynamic IPSS (DIPSS) (Passamonti et al., 2010), and DIPSS plus (Gangat et al., 2010) were evaluated for estimated survival.

\section{Statistical analysis}

The demographic data, clinical characteristics, and laboratory results were analyzed by descriptive analysis. Comparison of continuous variables was analyzed by independent t-test and categorical variables by Chisquare test. These variables included signs, symptoms, laboratory data, and results of JAK2V617F mutation that were analyzed in order to determine their relationship with complications. The significance of differences was determined if $P$ value was less than 0.05 . The variables that had a $P$ value less than 0.05 from univariate analysis were analyzed by backward selection as the multivariate analysis for determining the independent risk factors of thrombosis and bleeding. All data were analyzed using the SPSS version 16.0.

\section{Results}

\section{Demographic data}

There were $217 \mathrm{Ph}$-negative MPNs patients during the period of study. One hundred and fifty seven patients were diagnosed with PV, ET, or PMF and were included in this study. The reasons of exclusion were other hematologic diseases (six patients with thalassemia and two patients with myelodysplastic syndrome) and inadequate data (52 patients). Participants were classified to PV, ET and PMF as 68, 83 and 6 patients with the median age of 60 (range 18-88), 61 (range 21-89), and 68 (range 52-78) years respectively. ET was more prevalent in females but $\mathrm{PV}$ and PMF occurred more in males (male: female ratios were $32: 51,46: 22$, and 4:2, respectively).

\section{Clinical manifestations}

Table 1 shows clinical characteristics of ET, PV, and PMF patients. Asymptomatic cases were found in 54\% of ET and $30.8 \%$ of PV but none in PMF. The most common signs and symptoms in ET were splenomegaly $(10.8 \%)$, hepatomegaly $(8.4 \%)$, and dizziness $(7.2 \%)$. PV patients presented with splenomegaly (11.8\%), erythromelalgia, and facial plethora (both $10.3 \%$ ) whereas PMF had splenomegaly (100\%), hepatomegaly (50\%), and constitutional symptoms (50\%).

Twenty nine percent of PV patients presented with thrombosis which was more prevalent in ET and PMF (14.4\% and $0 \%$, respectively). Arterial thrombosis was more common than venous thrombosis with the common sites of ischemic stroke (22\%) and myocardial infarction (4.4\%). In ET, the common sites included critical limb ischemia (4.8\%), portal vein and cerebral sinus thrombosis (both 2.4\%).

Bleeding complications were found to occur $10.8 \%$ and $7.4 \%$ in ET and PV, respectively primarily manifesting with gastrointestinal hemorrhage. In PMF, one patient $(16.7 \%)$ had cutaneous bleeding. 
Clinical Manifestations and Risk Factors for Complications of Philadelphia Chromosome-Negative MPNs

\section{Laboratory data and JAK2V617F mutation}

The complete blood count and JAK2V617F mutation results of Ph-negative MPNs patients are shown in Table 1. The mean platelet count of ET patients was 1,191 x $10^{9} / \mathrm{L}$ which was higher than PV and PMF. The mean hemoglobin and hematocrit results of PV patients were $19.2 \mathrm{~g} / \mathrm{dl}$ and $58.2 \%$, respectively which was higher than ET and PMF. In PMF patients, all patients had anemia with a mean hemoglobin of $8.2 \mathrm{~g} / \mathrm{dl}$ and hematocrit of $25.3 \%$. A wide range of $\mathrm{WBC}$ and platelet counts were found in PMF with mean WBC count of $37 \times 10^{9} / \mathrm{L}$ (range $\left.3.1-104 \times 10^{9} / \mathrm{L}\right)$ and mean platelet count of $259 \times 109 / \mathrm{L}$ (range $28.8-991 \times 10^{9} / \mathrm{L}$ ). Some patients were analyzed for JAK2V617F mutation. The majority of cases harbored this mutation with $87.5 \%$ in PV, $69 \%$ in ET, and $100 \%$ in PMF.

\section{Risk factors of thrombosis}

The multivariate analysis of risk factors of thrombosis in ET is shown in Table 2. Patients with higher hemoglobin

Table 1. Clinical Characteristics and Laboratory Findings of ET, PV, and PMF Patients

\begin{tabular}{|c|c|c|c|c|}
\hline Clinical Characteristics & & $\begin{array}{c}\text { ET } \\
\text { No. }(\%)\end{array}$ & $\begin{array}{c}\text { PV } \\
\text { No. }(\%)\end{array}$ & $\begin{array}{c}\text { PMF } \\
\text { No. }(\%)\end{array}$ \\
\hline Total Patients (number) & & 83 & 68 & 6 \\
\hline Age (year) & & $61.1(21-89)$ & $59.6(18-88)$ & $68.3(52-78)$ \\
\hline Sex (male: female) & & $32: 51: 00$ & $46: 22(23 / 11)$ & $4: 02$ \\
\hline \multirow[t]{10}{*}{ Symptoms and signs } & Asymptomatic & $45(54.2)$ & $21(30.9)$ & 0 \\
\hline & Constitutional Symptoms & $1(1.2)$ & 0 & $3(50)$ \\
\hline & Dizziness & $6(7.2)$ & $5(7.4)$ & 0 \\
\hline & Fatigue & $5(6.02)$ & 0 & $2(33.3)$ \\
\hline & Headache & $1(1.2)$ & $3(4.4)$ & 0 \\
\hline & Seizure & $1(1.2)$ & 0 & 0 \\
\hline & Erythromelalgia & $1(1.2)$ & $7(10.3)$ & 0 \\
\hline & Facial Plethora & 0 & $7(10.3)$ & 0 \\
\hline & Hepatomegaly & $7(8.4)$ & $2(2.9)$ & $3(50)$ \\
\hline & Splenomegaly & $9(10.8)$ & $8(11.8)$ & $6(100)$ \\
\hline \multirow[t]{11}{*}{ Thrombotic Events (total) } & & $12(14.4)$ & $22(29.6)$ & 0 \\
\hline & Critical Limb Ischemia & $4(4.8)$ & 0 & 0 \\
\hline & Cerebral Infarction & $1(1.2)$ & $15(22.1)$ & 0 \\
\hline & Myocardial Infarction & $1(1.2)$ & $3(4.4)$ & 0 \\
\hline & Superior Mesenteric Artery & 0 & $1(1.5)$ & 0 \\
\hline & Pulmonary Embolism & 0 & $1(1.5)$ & 0 \\
\hline & Jugular Vein Thrombosis & 0 & $1(1.5)$ & 0 \\
\hline & Deep Vein Thrombosis & $1(1.2)$ & 0 & 0 \\
\hline & Portal Vein Thrombosis & $2(2.4)$ & 0 & 0 \\
\hline & Splenic Vein Thrombosis & 0 & $1(1.5)$ & 0 \\
\hline & Cerebral Sinus Thrombosis & $2(2.4)$ & 0 & 0 \\
\hline \multirow[t]{6}{*}{ Bleeding Events (total) } & & $9(10.8)$ & $5(7.4)$ & $1(16.67)$ \\
\hline & Cerebral Hemorrhage & $1(1.2)$ & $2(2.9)$ & 0 \\
\hline & Gastrointestinal Hemorrhage & $5(6)$ & $3(4.4)$ & 0 \\
\hline & Prolonged Postop Bleeding & $1(1.2)$ & 0 & 0 \\
\hline & Muscle & $1(1.2)$ & 0 & 0 \\
\hline & Skin & $1(1.2)$ & 0 & $1(16.67)$ \\
\hline \multirow[t]{3}{*}{ IPSET-thrombosis Score } & High & $42(50.6)$ & & \\
\hline & Intermediate & $20(24.1)$ & & \\
\hline & Low & $21(25.3)$ & & \\
\hline \multirow[t]{3}{*}{ International Study of PV Score } & High & & $1(1.5)$ & \\
\hline & Intermediate & & $33(48.5)$ & \\
\hline & Low & & $34(50)$ & \\
\hline \multirow{4}{*}{$\begin{array}{l}\text { Survival Rate Score } \\
\text { (IPSS/ DIPSS/ DIPSS Plus) }\end{array}$} & High & & & $2 / 2 / 03$ \\
\hline & Intermediate- 2 & & & $2 / 3 / 02$ \\
\hline & Intermediate-1 & & & $2 / 1 / 01$ \\
\hline & Low & & & - \\
\hline Laboratory Findings & & Mean (range) & Mean (range) & Mean (range) \\
\hline Hemoglobin $(\mathrm{g} / \mathrm{dl})$ & & $11.6(4-18.5)$ & $19.2(15-22)$ & $8.2(6-11)$ \\
\hline Hematocrit (\%) & & $36.1(13-59.8)$ & $58.2(42-69)$ & $25.3(19-33)$ \\
\hline White Blood Cell (x 109/L) & & $17.2(4-62.5)$ & $25(5.1-42)$ & $37.1(3.1-104)$ \\
\hline Platelet (x 109/L) & & $1,191(517-2,722)$ & $655(136-1,500)$ & $259(28.8-991)$ \\
\hline$J A K 2$ V617F Mutation & & No. $(\%)$ & No. $(\%)$ & No. $(\%)$ \\
\hline \multirow{2}{*}{ (in analyzed patients) } & Positive & $47(69.1)$ & $49(87.5)$ & $2(100)$ \\
\hline & Negative & $21(30.8)$ & $7(12.5)$ & $0(0)$ \\
\hline
\end{tabular}

ET: Essential thrombocythemia, PV: Polycythemia vera, PMF: Primary myelofibrosis, IPSET-thrombosis: International Prognostic Score of Thrombosis in World Health Organization-Essential Thrombocythemia, IPSS: International Prognostic Scoring System, DIPSS: Dynamic International Prognostic Scoring System 


\begin{tabular}{|c|c|c|c|c|}
\hline Variables & Odds Ratio & SD & P-value & $95 \% \mathrm{CI}$ \\
\hline \multicolumn{5}{|l|}{ Risk Factors of Thrombosis in ET } \\
\hline Male & 0.25 & 0.24 & 0.15 & $0.04-1.67$ \\
\hline Cardiovascular Risk Factors & 0.05 & 0.07 & $0.002 *$ & $0.005-0.58$ \\
\hline Hemoglobin & 4.72 & 3.69 & $0.047 *$ & $1.02-21.8$ \\
\hline Positive $J A K 2$ V617F & 0.16 & 0.28 & 0.29 & $0.006-4.68$ \\
\hline IPSET-thrombosis (Intermediate vs High risk) & 0.91 & 0.91 & 0.92 & $0.13-6.43$ \\
\hline \multicolumn{5}{|l|}{ Risk Factors of Thrombosis in PV } \\
\hline Age & 0.93 & 0.03 & $0.047 *$ & $0.87-0.99$ \\
\hline Splenomegaly & 0.09 & 0.14 & 0.11 & $0.005-1.70$ \\
\hline Plethora & 0.21 & 0.25 & 0.19 & $0.02-2.21$ \\
\hline Positive $J A K 2$ V617F Mutation & 0.33 & 0.27 & 0.17 & $0.07-1.61$ \\
\hline Risk Score of International Study of PV (Low vs Intermediate Risk) & 5.79 & 4.93 & $0.04 *$ & $1.09-30.7$ \\
\hline \multicolumn{5}{|l|}{ Risk Factors of Bleeding in ET } \\
\hline Age & 1.04 & 0.03 & 0.2 & $0.98-1.10$ \\
\hline Male & 2.87 & 2.23 & 0.18 & $0.62-13.2$ \\
\hline Constitutional Symptoms & 0.004 & 0.01 & 0.01 & $0.00-0.33$ \\
\hline Splenomegaly & 7.46 & 6.6 & $0.02 *$ & $1.32-42.2$ \\
\hline \multicolumn{5}{|l|}{ Risk Factors of Bleeding in PV } \\
\hline Age & 1.04 & 0.08 & 0.57 & $0.90-1.21$ \\
\hline Constitutional Symptoms & 2.93 & 0 & $0.01 *$ & $1.13 \mathrm{e}-10-0.08$ \\
\hline Hemoglobin & 1.24 & 0.14 & 0.05 & $0.999-1.542$ \\
\hline Platelet count & 1.01 & 0.002 & $0.03 *$ & $1.00-1.01$ \\
\hline Risk Score of International Study of PV (Low vs Intermediate Risk) & 0.23 & 0.41 & 0.42 & $0.01-8.00$ \\
\hline
\end{tabular}

* Statistical significance; ET: Essential thrombocythemia, PV: Polycythemia vera, SD: Standard deviation, CI: Confidence interval, IPSET-thrombosis: International Prognostic Score of Thrombosis in World Health Organization-Essential Thrombocythemia

levels had an increased thrombotic risk $[\mathrm{OR}=4.72$; $\mathrm{p}=0.047(95 \%$ CI $1.02-21.8)]$ whereas the presence of cardiovascular risk factors decreased the risk of thrombosis [OR=0.05; $\mathrm{p}=0.002(95 \% \mathrm{CI}$ 0.005-0.58)]. Sex and $J A K 2 \mathrm{~V} 617 \mathrm{~F}$ mutations were not statistically significant in association with thrombosis. For the IPSET-thrombosis score, this study compared only the intermediate and high risk groups since only one patient in the low risk group had thrombosis. The result showed IPSET-thrombosis also had no statistical significance associated with thrombotic risk $[\mathrm{OR}=0.91 ; \mathrm{p}=0.92(95 \% \mathrm{CI}$ 0.13-6.43)].

For the risk factors of thrombosis in PV (Table 2), the multivariate analysis revealed that the survival risk scores from International Study of PV (Tefferi et al., 2013) were associated with a risk of thrombosis $[\mathrm{OR}=5.79$; $\mathrm{p}=0.04$ (95\%CI 1.09-30.7)] when comparing low and intermediate risks. Only one patient in the high risk group had thrombosis and could not be analyzed. Older age patients showed a slightly decreased risk of thrombosis [OR=0.93; $\mathrm{p}=0.047$ (95\%CI 0.87-0.99)]. Splenomegaly, plethora, and $J A K 2 \mathrm{~V} 617 \mathrm{~F}$ mutation had no statistically significant association with thrombosis.

\section{Risk factors of bleeding}

Table 2 shows multivariate analysis of risk factors of bleeding in ET and PV, respectively. Splenomegaly was associated with a higher risk of bleeding [OR 7.46; $\mathrm{p}=0.02(95 \% \mathrm{CI} 1.32-42.2)]$ in ET. In PV, constitutional symptoms and higher platelet counts increased the risk of bleeding [OR 2.93; p=0.01 (95\%CI 1.13e-10-0.08) and OR $1.01 ; \mathrm{p}=0.03$ (95\%CI 1.00-1.01), respectively]. Higher hemoglobin levels tended to increase the bleeding risk without statistical significance. This study did not evaluate risk factors of thrombosis and bleeding in PMF due to the low number of patients.

\section{Other complications}

During follow up, one of the ET patients (1.2\%) transformed to acute myelogenous leukemia. Thrombotic complications occurred in five PV and ET patients. Two cases developed critical limb ischemia (one ET and one PV patients at 33 and 19 months, respectively) and three cases developed cerebral infarction (two with ET at 64 and 118 months and one PV patient at 14 months) even though all five cases received antiplatelet and cytoreductive agents. Neither secondary myelofibrosis nor death cases were reported.

\section{Discussion}

This study showed clinical manifestations of Thai Ph-negative MPN patients. For ET, the mean age was 61 years with minimum age of 18 years which were slightly higher than in the IPSET-thrombosis study (Barbui et al., 2012) (54.4 years and 14.8 years, respectively). Males found to be less than females which was similar to the IPSET-survival study (Passamonti et al., 2012). Common clinical manifestations included dizziness, hepatomegaly, splenomegaly, as well as thrombosis which occurred in $14.4 \%$ of the ET patients at diagnosis. Thrombotic events were close to those in the IPSET-thrombosis study (12\%) (Barbui et al., 2012). The mean platelet count in this study was higher than IPSET-thrombosis study $\left(1,191 \times 10^{9} / \mathrm{L}\right.$ versus $\left.771 \times 10^{9} / \mathrm{L}\right)$. Extreme thrombocytosis or a platelet count more than $1,000 \times 10^{9} / \mathrm{L}$ was reported to decrease the risk of arterial thrombosis (Barbui et al., 2012), however this does not explain the higher incidence of thrombosis in 
Clinical Manifestations and Risk Factors for Complications of Philadelphia Chromosome-Negative MPNs

this study. JAK2V617F mutation was found in $62 \%$ of ET patients which was similar with results from other studies (Klampfl et al., 2013; Tefferi et al., 2014) including those from Thailand (Kunnim and Auewarakul, 2010).

For risk factors of thrombosis in ET, this study showed that a higher hemoglobin was associated with an increased risk of thrombosis. It is possible that the patients with higher hemoglobin may have "masked PV" (Barbui et al., 2014) and then have higher risk of thrombosis compared with ET (Tefferi et al., 2013; Carobbio et al., 2011). This finding supports the proposed diagnostic WHO criteria for PV which was a decreased level of hemoglobin to $16.5 \mathrm{~g} / \mathrm{dl}$ in males and $16 \mathrm{~g} / \mathrm{dl}$ in females along with a careful interpretation of bone marrow biopsy (Tefferi et al., 2014). When compared with the IPSET-thrombosis score, this study showed many different results not only the risk of higher hemoglobin, but also the protective factors for cardiovascular risk. It was also contrast with study from Turkey that found higher dyslipidemia patients in ET and PV with thrombosis (Sag et al., 2015). The hypothesis was that some patients that have cardiovascular risk factors already received medications for prevention of thrombosis. The non-statistically significant prognosis of $J A K 2 \mathrm{~V} 617 \mathrm{~F}$ mutation as well as the IPSET-thrombosis score in this study may be explained by the low numbers and thrombotic events of the patients.

For PV patients, the demographic data from this study was similar to the International Study of PV (Tefferi et al., 2013) with a mean age of 59.6 years versus 61 years, respectively with the minimal age being 18 years in both studies. Male sex was about two times more prevalent than females compared to the 1:1 ratio in the International Study. Eighty seven percent of PV patients harbored the $J A K 2 \mathrm{~V} 617 \mathrm{~F}$ mutation which was slightly lower than previous studies (Tefferi et al., 2008; Tefferi et al., 2013; Klampfl et al., 2013). Thrombosis was found to be $29 \%$ which was slightly higher than International Study (23.4\%) and higher than ET. The score that predicted survival from the International Study of PV was significantly associated with thrombosis. However, age more than 65 years which was a risk factor from the prospective European Collaboration on Low-dose Aspirin in Polycythemia Vera (ECLAP) study (Marchioli et al., 2005), were not associated with thrombotic risk in this study.

For risk factors of bleeding, splenomegaly was associated with this complication in ET whereas the large international study revealed previous hemorrhage and aspirin as independent risk factors (Finazzi1 et al., 2012). There was no clear explanation about this finding, These patients might have pre-fibrotic PMF that increased their risk of bleeding compared to WHO-defined ET (Finazzi1 et al., 2012). A higher platelet count was associated with bleeding in PV. This was supported by previous studies (Budde U and van Gendernen PJ, 1997) that reported that extreme thrombocytosis (platelet more than 1,000 $\mathrm{x} 109 / \mathrm{L}$ ) was associated with bleeding. Analysis from a prospective PT1 study in ET showed that a platelet count outside of normal range was associated with major hemorrhage (Campbell et al., 2012). However, our study did not demonstrate this correlation in ET patients.

There were only six PMF patients included in this study with a mean age of 68 years which was close to 65 years from the large international cohort study (Gangat et al., 2010). The most common clinical manifestations were splenomegaly, hepatomegaly, constitutional symptoms, fatigue that differed from ET and PV. All patients had anemia with a wide range of WBC counts and platelet counts. Due to the low numbers of PMF patients, this study could not determine the value of the international prognostic score such as IPSS, DIPSS, and DIPSS plus.

During follow up, five patients with ET and PV had thrombotic complications even though they received antiplatelet and cytoreductive treatment which supported the high risk of thrombosis in these groups of $\mathrm{Ph}$-negative MPNs. Leukemic transformation was found only in one ET patient. Neither secondary myelofibrosis nor death was reported possibly due to short follow-up time and some patients were lost to follow-up.

This study provided more information about clinical manifestations and complications of Ph-negative MPNs in Thailand. The new international prognosis scores such as IPSET-thrombosis and risk scores from International Study of PV were used in the analysis. The limitations of this study were that it was a retrospective study that resulted in incomplete data, unconfirmed diagnosis of $\mathrm{Ph}$ negative MPNs according to current WHO Classification, low numbers of patients, and some patients were not evaluated for JAK2V617F mutation.

In conclusion, Ph-negative MPNs had varied clinical manifestations. JAK2V617F mutations were present in the majority of PV, ET, and PMF patients $(87,69$, and $100 \%$, respectively). This study confirmed that thrombosis (29\% in PV, $14 \%$ in ET) and bleeding (16\% in PMF, $10 \%$ in ET, and $7 \%$ in PV) were the most significant complications in the patients with Ph-negative MPN. .

\section{References}

Barbui T, Barosi G, Birgegard G, et al (2011). Philadelphianegative classical myeloproliferative neoplasms: critical concepts and management recommendations from European LeukemiaNet. J Clin Oncol, 29, 761-70.

Barbui T, Finazzi G, Carobbio A, et al (2012). Development and validation of an international prognostic score of thrombosis in world health organization-essential thrombocythemia (IPSET-thrombosis). Blood, 120, 5128-33.

Barbui T, Thiele J, Gisslinger H, et al (2014). Masked polycythemia vera $(\mathrm{mPV})$ : results of an international study. Am J Hematol, 89, 52-4.

Budde U, van Gendernen PJ (1997). Acquired von Willebrand disease in patients with high platelet counts. Thromb Hemost, 23, 425-31.

Campbell PJ, MacLean C, Beer PA, et al (2012). Correlation of blood counts with vascular complications in essential thrombocythemia: analysis of the prospective PT1 cohort. Blood, 120, 1409-11.

Carobbio A, Thiele J, Passamonti F, et al (2011). Risk factors for arterial and venous thrombosis in WHO-defined essential thrombocythemia: An international study of 891 patients. Blood, 117, 5857-9.

Cervantes F, Dupriez B, Pereira A, et al (2009). New prognostic scoring system for primary myelofibrosis based on a study of the International Working Group for Myelofibrosis Research and Treatment. Blood, 113, 2895-901. 
Finazzi1 G, Carobbio A, Thiele J, et al (2012). Incidence and risk factors for bleeding in 1104 patients with essential thrombocythemia or prefibrotic myelofibrosis diagnosed according to the 2008 WHO criteria. Leukemia, 26, 716-9.

Gangat N, Caramazza D, Vaidya R, et al (2010). DIPSS Plus: A refined Dynamic International Prognostic Scoring System for primary myelofibrosis that incorporates prognostic information from karyotype, platelet count, and transfusion status. J Clin Oncol, 29, 392-7.

Klampfl T, Gisslinger H, Harutyunyan AS, et al (2013). Somatic mutations of Calreticulin in myeloproliferative neoplasms. $N$ Engl J Med, 369, 2379-90.

Kunnim S, Auewarakul J (2010). JAK2 mutation in myeloproliferative neoplasms. J Hematol Transfus Med, 1, 55-62.

Marchioli R, Finazzi G, Landolfi R, et al (2005). Vascular and neoplastic risk in a large cohort of patients with polycythemia vera. J Clin Oncol, 23, 2224-32.

Passamonti F, Cervantes F, Maria A, et al (2010). A dynamic prognostic model to predict survival in primary myelofibrosis: a study by the IWG-MRT (InternationalWorking Group for Myeloproliferative Neoplasms Research and Treatment). Blood, 115, 1703-8.

Passamonti F, Thiele J, Girodon F, et al (2012). A prognostic model to predict survival in 867 World Health Organizationdefined essential thrombocythemia at diagnosis: a study by the International Working Group on Myelofibrosis Research and Treatment. Blood, 120, 1197-201.

Sag SM, Gorukmez O, Ture M, et al (2015). MMP2 Gene$735 \mathrm{C} / \mathrm{T}$ and MMP9 gene $-1562 \mathrm{C} / \mathrm{T}$ polymorphisms in $J A K 2 \mathrm{~V} 617 \mathrm{~F}$ positive myeloproliferative disorders. Asian Pac J Cancer Prev, 16, 443-9.

Tefferi A, Vardiman JW (2008). Classification and diagnosis of myeloproliferative neoplasms: the 2008 World Health Organization criteria and proposed diagnosis algorithm. Leukemia, 22, 14-22.

Tefferi A, Rumi E, Finazzi G, et al (2013). Survival and prognosis among 1545 patients with contemporary polycythemia vera: An international study. Leukemia, 27, 1874-81.

Tefferi A, Guglielmelli P, Larson DR, et al (2014). Long-term survival and blast transformation in molecularly annotated essential thrombocythemia, polycythemia vera, and myelofibrosis. Blood, 124, 2507-13.

Tefferi A, Thiele J, Vannucchi AM, et al (2014). An overview on $C A L R$ and $C S F 3 R$ mutations and a proposal for revision of WHO diagnostic criteria for myeloproliferative neoplasms. Leukemia, 28, 1407-13. 